\title{
Numerical Exploration of the RI/MOM Scheme Gauge Dependence*
}

\author{
L. Giusti ${ }^{\text {ab }}$, S. Petrarca ${ }^{\text {cd }}$, B. Taglienti ${ }^{\mathrm{d}}$ and N. Tantalo ${ }^{\text {ef }}$ \\ a Theory Division, CERN, CH-1211 Geneva 23, Switzerland \\ ${ }^{\mathrm{b}}$ Centre de Physique Théorique, CNRS Luminy, Case 907, F-13288 Marseille Cedex 9, France \\ cDipartimento di Fisica, Università di Roma "La Sapienza", P.le A. Moro 2, I-00185 Rome, Italy \\ ${ }^{\mathrm{d} I N F N}$ Roma 1, P.le A. Moro 2, I-00185 Rome, Italy \\ eDipartimento di Fisica, Università di Roma "Tor Vergata", V. R. Scientifica 1, I-00133 Rome, Italy \\ ${ }^{\mathrm{f}}$ INFN Roma 2, V. R. Scientifica 1, I-00133 Rome, Italy
}

\begin{abstract}
The gauge dependence of some fermion bilinear RI/MOM renormalization constants is studied by comparing data which have been gauge-fixed in two different realizations of the Landau gauge and in a generic covariant gauge. The very good agreement between the various sets of results and the theory indicates that the numerical uncertainty induced by the lattice gauge-fixing procedure is below the statistical errors of our data sample which is of the order of $(1 \div 1.5) \%$.
\end{abstract}

\section{Introduction and Strategy}

Non-perturbative renormalization techniques [1:2] have become a crucial ingredient in lattice computations of fundamental QCD parameters and hadronic matrix elements. The RI/MOM non-perturbative renormalization proposed in Refs. [3:1] has been successfully applied to compute renormalization constants of composite fermion operators in many lattice regularizations 3, 3, 1, 5, 6.,7]. The method imposes renormalization conditions on conveniently defined amputated Green functions computed nonperturbatively between off-shell quark states at large virtuality in a fixed gauge:

$\left.Z_{\Gamma}\left\langle p\left|O_{\Gamma}\right| p\right\rangle\right|_{p^{2}=\mu^{2}}=\left\langle p\left|O_{\Gamma}\right| p\right\rangle_{0}$.

The renormalization constants depend in general on the external states and therefore on the gauge in which the renormalization conditions have been imposed. For gauge-invariant operators the coefficients needed to match in a given gauge-invariant scheme has to cancel these dependences up to higher orders in the continuum perturbative expansion and up to discretization

\footnotetext{
${ }^{*}$ Talk given at Lattice 2002 by N.T.
}

errors.

The existence of both continuum and lattice Gribov copies and the numerical noise that they can generate (for a recent review see Ref. [8]) is an unsolved problem of the lattice non-perturbative gauge fixing. The real concern is, of course, the influence that these phenomena may have on the values of the renormalization constants, when computed using non-gauge-invariant quantities as in the RI/MOM scheme.

Here we discuss the results of a numerical study of the systematics induced by the gauge-fixing procedure on the RI/MOM determinations of the quark field $\left(Z_{\psi}\right)$, the axial-vector $\left(Z_{A}\right)$ and of the scalar density $\left(Z_{S}\right)$ renormalization constants (for more details see Ref. [9]). We have done this by imposing the standard lattice Landau gauge and another realization of the Landau condition (Landau1 gauge). Whereas the two realizations impose the same gauge-fixing condition in the naive continuum limit, they are affected by different Gribov ambiguities. By comparing the different sets of results for the renormalization constants, we have found differences which are negligible within our statistical errors (of the order of $(1 \div 1.5) \%)$. 
An interesting feature of the Landau1 gauge is that it can be generalized to impose a generic covariant gauge on the lattice, as proposed in Ref. 10. By exploiting this opportunity, we have performed an exploratory study of the gauge dependence of off-shell Green functions measured in a generic covariant gauge.

\section{Results and Discussion}

We have used a sample of $80 \mathrm{SU}(3)$ gauge configurations retrieved from the repository at the "Gauge Connection" (http://qcd.nersc.gov/), which were generated with the Wilson gluonic action at $\beta=6.0$ and $V \times T=16^{3} \times 32$. By using the discretized version (see [10] for details) of the following functionals:

$F_{A}[G]=\left\|A^{G}\right\|^{2}=\int d^{4} x \operatorname{Tr}\left[A_{\mu}^{G} A_{\mu}^{G}\right]$

$H_{A}[G]=\int d^{4} x \operatorname{Tr}\left[\left(\partial_{\mu} A_{\mu}^{G}\right)^{2}\right]$

$H_{(A, \Lambda)}[G]=\int d^{4} x \operatorname{Tr}\left[\left(\partial_{\mu} A_{\mu}^{G}-\Lambda\right)^{2}\right]$,

we have rotated each configuration in the Landau gauge, in the Landau1 gauge and in the covariant gauge. The function $\Lambda$ belongs to the Lie algebra of the group and has been generated with a gaussian distribution corresponding to the value $\xi=8$ of the bare gauge fixing parameter.

Once the quark propagator $\mathcal{S}(x, 0)$ has been computed for each $\mathrm{SU}(3)$ configuration and gauge and Fourier-transformed, we have determined the inverse Euclidean bare-quark propagator $\mathcal{S}^{-1}(p a)$. We have computed the amputated Green functions of the local quark bilinears $O_{\Gamma}(x)=\bar{\psi}(x) \Gamma \psi(x)$ in the Fourier space (in what follows we adopt the conventions of Ref. [11])

$\Lambda_{\Gamma}(p a)=\mathcal{S}^{-1}(p a) G_{\Gamma}(p a) \mathcal{S}^{-1}(p a)$,

where

$G_{\Gamma}(p)=\int d^{4} x d^{4} y e^{-i p(x-y)}\left\langle\psi(x) O_{\Gamma}(0) \bar{\psi}(y)\right\rangle$

and $\Gamma$ is a generic Dirac matrix corresponding to $\Gamma=\{\mathrm{A}, \mathrm{S}\}=\left\{\gamma_{\mu} \gamma_{5}, \mathbb{I}\right\}$. The renormalization

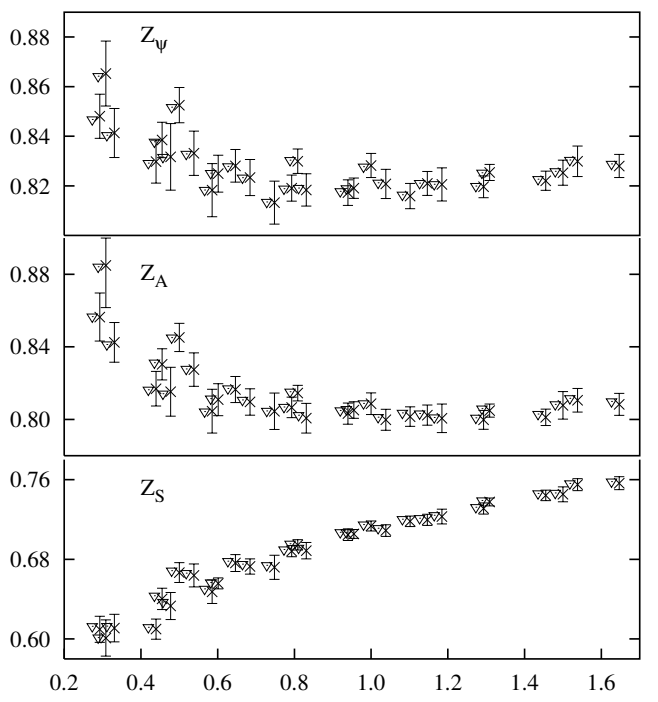

Figure 1. The $Z_{A}$ and $Z_{S}$ renormalization constants are shown as a function of $(a \mu)^{2}$ for the Landau (crosses) and Landau1 (triangles) gauges. The data have been slightly displaced in the $x$-direction and the error bars for one set only have been shown.

constant $Z_{\Gamma}^{\mathrm{RI}}\left(\mu a, g_{0}\right)$, which defines the renormalized operator $\hat{\mathcal{O}}_{\Gamma}^{\mathrm{RI}}=Z_{\Gamma}^{\mathrm{RI}} \mathcal{O}_{\Gamma}$, is fixed by imposing in the chiral limit the renormalization condition

$\left.Z_{\Gamma}^{\mathrm{RI}}(\mu a) Z_{\psi}^{-1}(\mu a) \operatorname{Tr} \mathrm{P}_{\Gamma} \Lambda_{\Gamma}(p a)\right|_{p^{2}=\mu^{2}}=1$,

where $\mathrm{P}_{\Gamma}$ is a suitable projector on the treelevel amputated Green function and where the $\mathrm{RI}$ wave-function renormalization constant $Z_{\psi}$ is defined as

$Z_{\psi}(\mu a)=\lim _{m \rightarrow 0}-i \frac{1}{12} \operatorname{Tr}\left[\frac{\partial S^{-1}(p a)}{\partial \not p}\right]_{p^{2}=\mu^{2}}$.

Even though $\mathcal{O}_{A}$ and $\mathcal{O}_{S}$ are gaugeindependent operators, their matrix elements between quark states are gauge dependent. In Fig. 1, the numerical results for $Z_{\psi}, Z_{A}$ and $Z_{S}$ calculated in the Landau and in the Landau1 gauge as a function of the square lattice momenta, are shown. Our data are in very good agreement with the results reported in the literature 11].

The results for all correlators, corresponding to Landau and Landau1 gauges, coincide within the statistical errors. As a consequence we can conclude that the data do not show any residual effect due to the presence of lattice Gribov's 

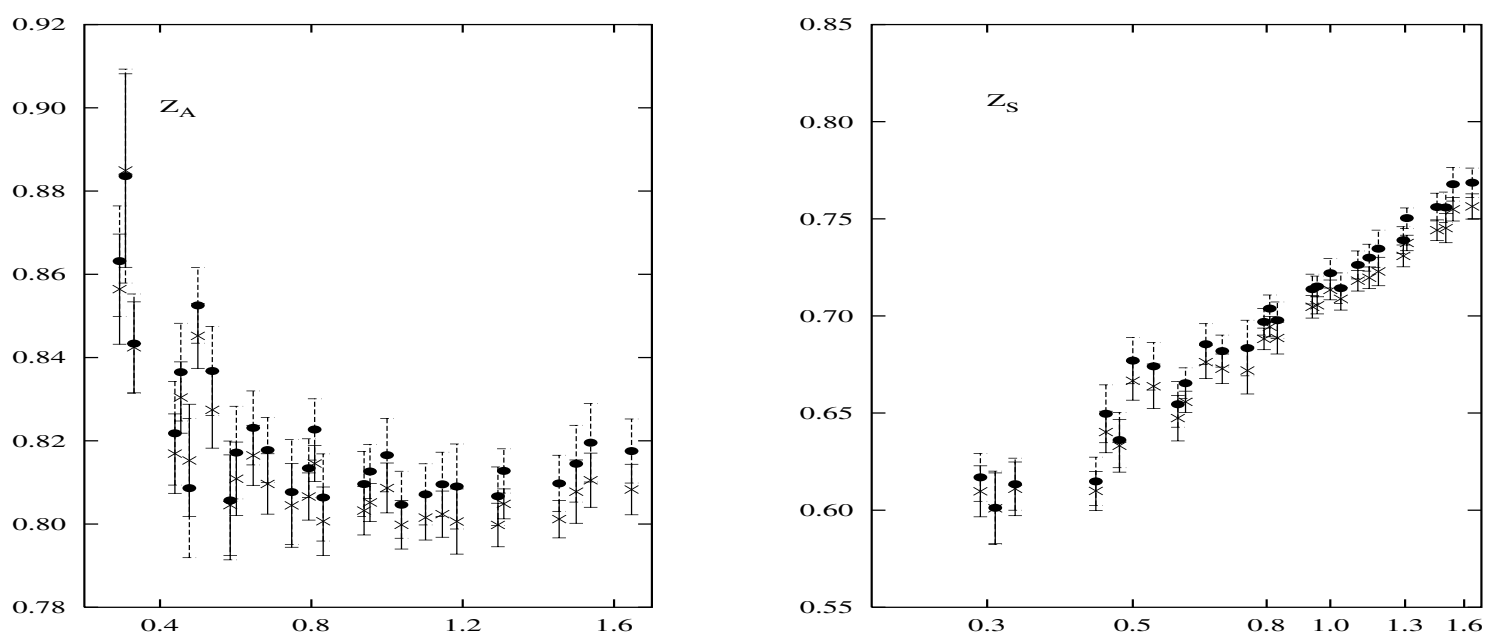

Figure 2. The behaviour of $Z_{A}$ and $Z_{S}$ is shown as a function of $(a \mu)^{2}$ for the Landau (black dots) and the covariant gauge (crosses) data. The residual gauge-dependence is of the order of $1.5 \%$

copies in the statistical sample of configurations generated and for the lattice used.

To show our sensitiveness to the choice of the gauge, we compare the results obtained in the Landau and in the covariant gauge with the bare gauge parameter fixed to $\xi=8$.

The primary quantities measured on the lattice, $Z_{\psi}, \Lambda_{A}$ and $\Lambda_{S}$, show a statistically significant gauge dependence (as you can see in Fig. 2 of Ref. [9]) while the renormalization constants $Z_{A}, Z_{S}$ shown in Fig. 2, obtained by computing the ratios as indicated in Eq. (7), could have at most a residual gauge dependence (of the order of $1.5 \%)$ which is not detectable within the statistical precision of our simulation. For these quantities, the fluctuations of the simulation hide the weak gauge dependence that is expected in perturbation theory from the next-to-leading order terms in $\alpha_{s}$.

These results indicate an upper limit to the numerical troubles that can be expected in the $\mathrm{RI} / \mathrm{MOM}$ renormalization constants because of the lattice gauge fixing.

\section{REFERENCES}

1. G. Martinelli, C. Pittori, C. T. Sachrajda, M. Testa and A. Vladikas, Nucl. Phys. B 445 (1995) 81.
2. K. Jansen et al., Phys. Lett. B 372 (1996) 275; M. Lüscher, S. Sint, R. Sommer and H. Wittig, Nucl. Phys. B 491 (1997) 344. S. Capitani, M. Lüscher, R. Sommer and H. Wittig [ALPHA Collaboration], Nucl. Phys. B 544 (1999) 669.

3. G. Martinelli, S. Petrarca, C. T. Sachrajda and A. Vladikas, Phys. Lett. B 311 (1993) 241 [Erratum,ibid. B 317 (1993) 660].

4. G. Martinelli, G. C. Rossi, C. T. Sachrajda, S. R. Sharpe, M. Talevi and M. Testa, Phys. Lett. B 411 (1997) 141.

G. Martinelli, G. C. Rossi, C. T. Sachrajda, S. R. Sharpe, M. Talevi and M. Testa, Nucl. Phys. B 611 (2001) 311.

5. S. Aoki et al. [JLQCD Collaboration], Phys. Rev. Lett. 82 (1999) 4392.

6. T. Blum et al., Phys. Rev. D 66 (2002) 014504

7. L. Giusti, C. Hoelbling and C. Rebbi, Phys. Rev. D 64 (2001) 114508. and Nucl. Phys. Proc. Suppl. 106 (2002) 739.

8. L. Giusti, M. L. Paciello, C. Parrinello, S. Petrarca and B. Taglienti, Int. J. Mod. Phys. A 16 (2001) 3487.

9. L. Giusti, S. Petrarca, B. Taglienti and N. Tantalo, Phys. Lett. B 541 (2002) 350

10. L. Giusti, M. L. Paciello, S. Petrarca and B. Taglienti, Phys. Rev. D 63 (2001) 014501.

11. V. Gimenez, L. Giusti, F. Rapuano and M. Talevi, Nucl. Phys. B 531 (1998) 429. 